\title{
Impedance Spectroscopy Analysis of Hydration in Ordinary Portland Cements Involving Chemical Mechanical Planarization Slurry
}

\author{
Jin-Ha Hwang ${ }^{\dagger}$ \\ Department of Materials Science and Engineering, Hongik University, Seoul 121-791, Korea
}

(Received March 28, 2012; Revised April 10, 2012; Accepted April 13, 2012)

\begin{abstract}
Impedance spectroscopy was used to monitor the hydration in the electrical/dielectric behaviors of chemical mechanical planarization (CMP)-blended cement mixtures. The electrical responses were analyzed using their equivalent circuit models, leading to the separation of the bulk and electrode based responses. The role of the CMP slurry was monitored as a function of the relative compositions of the CMP-blended cements, i.e. water, CMP slurry, and ordinary Portland cement. The presence of $\mathrm{Al}_{2} \mathrm{O}_{3}$ nanocrystals in the CMP slurries appeared to accelerate the hydration process, along with a more tortuous microstructure in the hydration, with enhanced hydration products. The frequency-dependent impedance spectroscopy was proven to be a highly efficient approach for evaluating the electrical/dielectric monitoring of the change in the pore structure evolution that occurs in CMP-blended cements.
\end{abstract}

Key words : Ordinary portland cements, CMP slurry, Hydration, Impedance spectroscopy, Electrical/dielectric properties

\section{Introduction}

C hemical mechanical planarization (CMP) is a possible core process in back-end semiconducting processing. After the application of CMP processing to the manufacturing steps, the CMP solutions possess considerable quantities of wastewater consisting of nanomaterial-containing sludge, which can be potentially dangerous if exposed to the environment. The CMP sludge can include over 200 types of organic and inorganic materials. ${ }^{1)}$ Thus, efficient control of the semiconductor wastewater for recovery, reuse, and recycling has attracted significant academic and industrial attention for the reuse of CMP-based materials. Lee et al. reported on CMP mortar safety issues, leading to the conclusion that the leaching concentration of heavy materials was far below that dictated by safety regulations and that their mechanical strength and physical features were equivalent or superior to their counterparts in ordinary Portland cements. $^{2)}$

The CMP process should require simultaneous mechanical abrasion and electrochemical dissolution in removing the patterned materials with the aim of achieving global planarization on large-scale Si wafers. For Cu-CMPs, the planarization step is divided into three steps: removal of the passive film, dissolution of the metal from the freshly exposed surface sites, and rapid repassivation of the freshly exposed metal. On copper surfaces, coarse insoluble prod-

Corresponding author : Jin-Ha Hwang

E-mail : jhwang@hongik.ac.kr

Tel : +82-2-320-3069 Fax : +82-2-333-0127 ucts, e.g. copper oxide and hydroxide in the form of $\mathrm{Cu}_{2} \mathrm{O}$, $\mathrm{CuO}$, and $\mathrm{Cu}(\mathrm{OH})_{2}$, are formed instead of the protective (passive) oxide film. Such nonmetallic materials are distributed in the CMP solvent materials. Therefore, the final solution is composed of the original CMP constituents and additional polishing residues. These contaminated materials should be recycled into useful material resources. Also, the entrapment of such nonmetallic materials should be connected with effective living products. Therefore, the current study proposes the entrapment of nonmetallic materials and their reuse as building blocks for domestic and industrial applications.

The increasing use of CMP materials has resulted in an increasing amount of processed materials, including high ceramic oxides in the nanoscale range. The planarization of these materials includes the metal lines, a barrier layer, and an interlayer of dielectric materials. The effective use of CMP materials is required in order to achieve environmentally benign processing cycles. The waste materials can lead to high processing costs if they are subjected to recycling and repurification. Thus, the entrapment of such material debris will be valuable in adopting the CMP process as a core processing step in semiconductor technology, because the solidified CMP materials can be used as everyday construction bricks for houses, office buildings, and other structures.

Cement-based materials have been investigated with regard to their hydration processes using mercury intrusion porosimetry, scanning electron microscopy, and BrunauerEmmett-Teller (BET) methods. These characterization tools exhibit inherent disadvantages in the reproducible data representation after monitoring the main features of the 
hydration process, and this is expected to result from the destructive features in the sample preparation and data analysis. Frequency-dependent impedance spectroscopy has also been proposed for in situ evaluation of hydration in cement-based materials: its non-destructive characteristics do not involve data acquisition interruptions and allow for long time measurements of the electrical properties during continuous setting. ${ }^{3-5)}$

Impedance spectroscopy (IS) is known to be a powerful tool for investigating the electrical/dielectric properties of medium resistivity materials. The frequency-dependent IS allows for the separation of the bulk-based responses from the electrode-related phenomena on the basis of a quantified evaluation of the electrical and dielectric information. ${ }^{6}$ Furthermore, the Nyquist plot depression analysis $\left(-Z_{\text {imag }}\right.$ versus $\mathrm{Z}_{\text {real }}$ ) can provide information on the inhomogeneity of the electrical origin components in microscale, which governs the corresponding bulk information. The electrical/ dielectric properties determined through hydration in cement-based materials have been reported from the ionic conduction among the highly resistive solid phases. ${ }^{3)}$ The ionic species are provided through dissolution of the ionic species of cement materials after reaction with the water reactants. The high fraction of the liquid phase provides conducting paths and therefore guarantees percolation through the paste. The corresponding electrical information is assumed to be explained by the equivalent circuits consisting of resistors and capacitors, and sometimes including inductive components.

The current study emphasizes the electrical monitoring of the effect of the CMP slurry on the hydration process that occur in cement-based materials using frequency-dependent IS. An alumina-based CMP slurry was chosen as a reference system in a series of CMP-blended cements. The CMPblended ordinary Portland cement (OPC) pastes should be investigated in the hydration of cement materials, for comparison with OPC pastes. The electrical/dielectric information was monitored through in situ impedance measurements in terms of hydration time and processing variables, e.g. the amount of water, humidity conditions, and powder preparation methods. The implications of the frequency-dependent IS are discussed with the aim of understanding the underlying hydration processes in CMP-blended cements.

\section{Experimental Procedures}

OPCs (Hyundai Cements, Korea) were sieved using a steel mesh of less than $150 \mu \mathrm{m}$. The sieved cement powder was blended with an alumina-based slurry where the $\mathrm{Al}_{2} \mathrm{O}_{3}$ particles had an average diameter of $130 \mathrm{~nm}$ in $5 \mathrm{wt} \%$, according to the analyzed composition provided in Table 1. The $\mathrm{pH}$ of the CMP slurry was maintained between 3 and 4 . Upon mixing the components (Table 1), tap water was used as the $\mathrm{H}_{2} \mathrm{O}$ source ingredient.

The components were weighed and are summarized in Table 1. The mixtures (or pastes) were hand-mixed for $10 \mathrm{~s}$,
Table 1. Sample Composition (in fractions) Employed in the CMP-slurry Mixtures

\begin{tabular}{cccc}
\hline Component & A1 & M1 & R1 \\
\hline Cement & 0.6 & 0.6 & 0.6 \\
Slurry & 0.4 & 0.3 & - \\
Water & & 0.1 & 0.4 \\
\hline
\end{tabular}

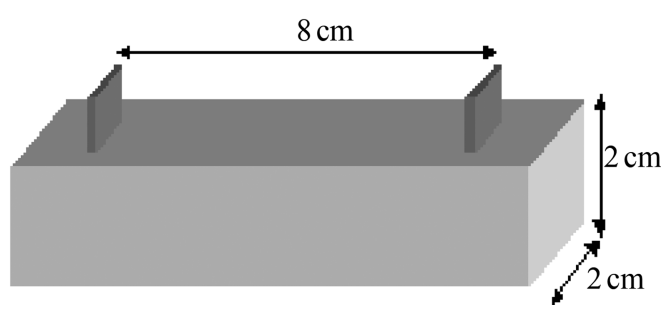

Fig. 1. Experimental setup for the impedance measurement employed in the CMP-blended cement mixtures in which the container was maintained under $100 \%$ relative humidity.

then poured into a mortar mixer, and mixed in speed sequences of $1 \mathrm{~min}$ at a low speed (62 RPM), $2 \mathrm{~min}$ at a high speed (125 RPM), and 15 min at a low speed (62 RPM). The mixtures were tapped in the container for $1 \mathrm{~min}$ and poured into the specimen fixtures (as shown in Fig. 1). In order to prevent bleeding and to ensure uniform states, the fixtures $(8 \mathrm{~cm} \times 2 \mathrm{~cm} \times 2 \mathrm{~cm})$ were made from polycarbonate plastics, where two planar electrodes were inserted into the pastes in the rectangular box, as shown in Fig. 1. The geometrical factor (i.e. the ratio of the length to the cross sectional area) was calculated to be $2 \mathrm{~cm}^{-1}$ with a relative error of $10 \%$ in specimen height. Epoxy was used as the sealing material to protect against water in order to prevent leakage through the barrier along the container wall. The two electrodes were connected to a frequency-response analyzer (SI-1260, Solartron, AMTEK, Inc., England). The impedance spectra were collected as a function of the frequency at 10 points per decade in a logarithmic manner between 10 $\mathrm{MHz}$ and $10 \mathrm{~Hz}$ using the ZPlot and ZView software (Scribner Associates Inc., USA). The measurement setup was maintained under $100 \%$ relative humidity in ambient conditions, where the sealed chamber incorporating the vessel was filled with tap water.

\section{Results and Discussion}

A typical impedance spectrum of an OPC cement is shown in Fig. 2. The impedance spectra were resolved into two regimes as a function of the measurement frequency: the high frequency region was assigned to the bulk-related responses and the low frequency portion was attributed to the polarization that occurs at the cement/electrode interfaces. The electrode-based responses reflect the sum of the two individual electrode effects adjacent to the cement-based materials because the current electrode configuration involves a two-point electrode. The impedance features enable equivalent circuits reflecting both bulk and electrode contributions, 


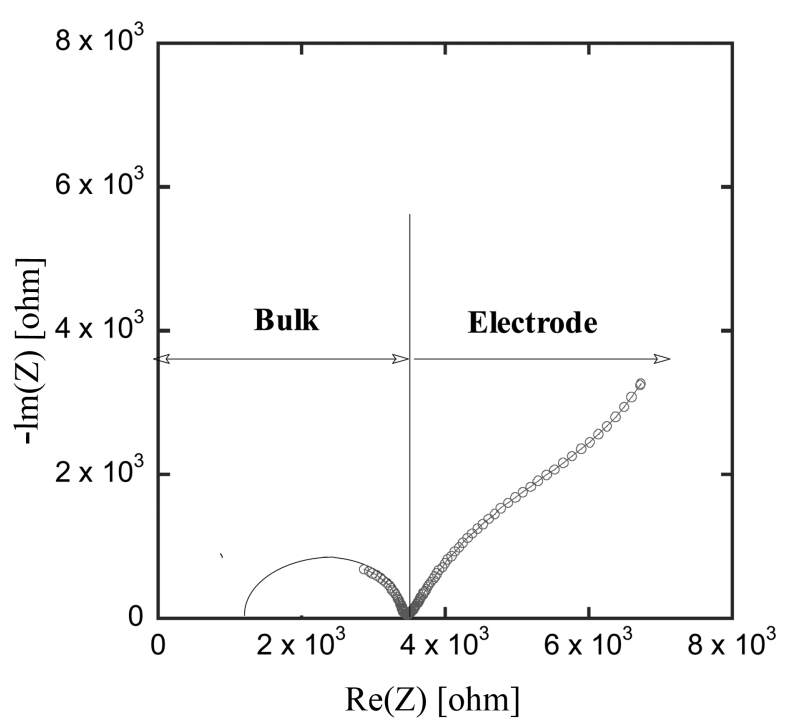

Fig. 2. Typical impedance spectra as a frequency where the frequency increases from right to left. The bulk and electrode rated responses are separated by the position of the cusp in the impedance spectra.

as shown in Fig. 3. The bulk and electrode responses were divided into bulk and electrode based responses, as shown in Fig. 2. The high frequency information reflects the bulk effect related to the hydration in the cements. The low frequency

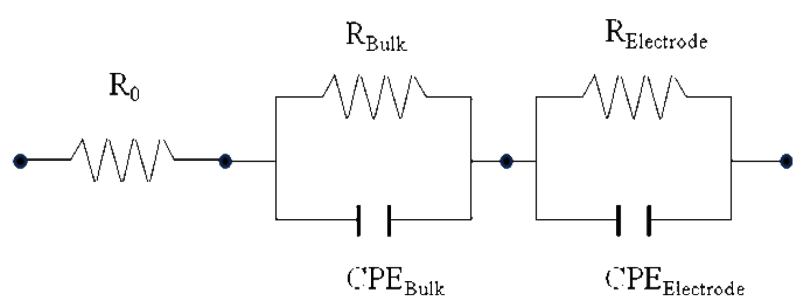

Fig. 3. Equivalent circuit model employed for the CMP-blended cement mixtures where $\mathrm{R}_{0}, \mathrm{R}_{\mathrm{Bulk}}$, and $\mathrm{CPE}_{\mathrm{Bulk}}$ are assigned to the bulk responses, and $\mathrm{R}_{\text {Electrode }}$ and $\mathrm{CPE}_{\text {Electrode }}$ to the electrode-related components. $\mathrm{R}_{0}$ reflects the high frequency intercept probably due to a partial portion in bulk responses composed of $R_{\text {Bulk }}$ and $\mathrm{CPE}_{\mathrm{Bulk}}$.

information indicates the polarization between ionic solution and electronic electrodes. According to the equivalent circuit description, the current impedance spectra are represented by $\mathrm{R}_{0}\left(\mathrm{R}_{\text {Bulk }} \mathrm{CPE}_{\text {Bulk }}\right)\left(\mathrm{R}_{\text {Electrode }} \mathrm{CPE}_{\text {Electrode }}\right)$, where the $\mathrm{R}$ and $\mathrm{CPE}$ (constant phase element) components are connected in parallel, but the overall networks are connected in series. Furthermore, CPE indicates a constant phase element involving the capacitance-like behavior of the arc depression. In the current circuit description, $\mathrm{R}_{0}$ reflects the high frequency intercept due to a partial portion in the bulk responses of the $\mathrm{R}_{\mathrm{Bulk}}$ and $\mathrm{CPE}_{\mathrm{Bulk}}$.

As shown in Fig. 4, the impedance spectra were collected

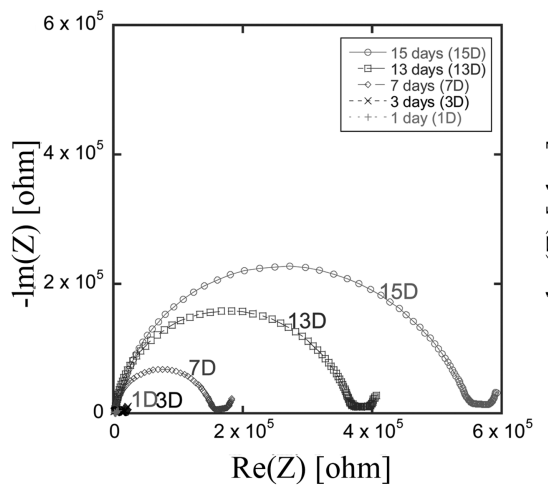

(a)

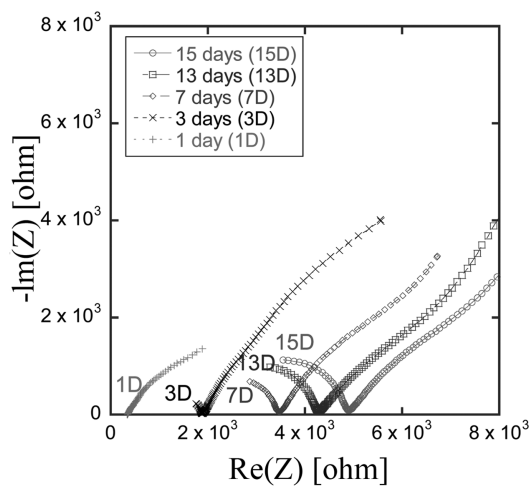

(c)

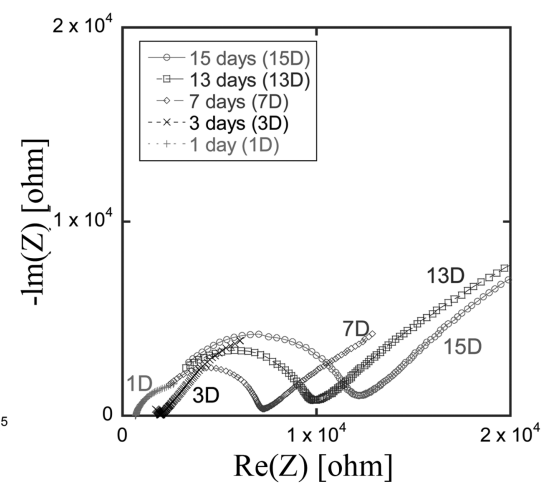

(b)

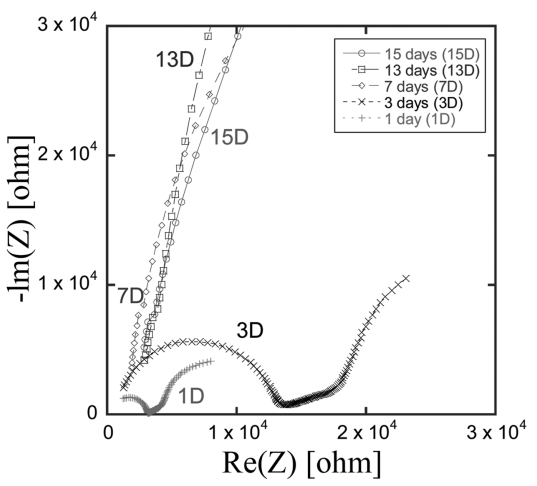

(d)

Fig. 4. Raw impedance spectra in the hydration of the OPC cements involving water and CMP slurry: (a) A1, (b) M1, and (c) R1 as a function of the hydration time. The impedance spectra were collected at $1,3,7$, and 15 day intervals. (d) Enlargement of Fig. 4(a) at an early stage of hydration in A1. 


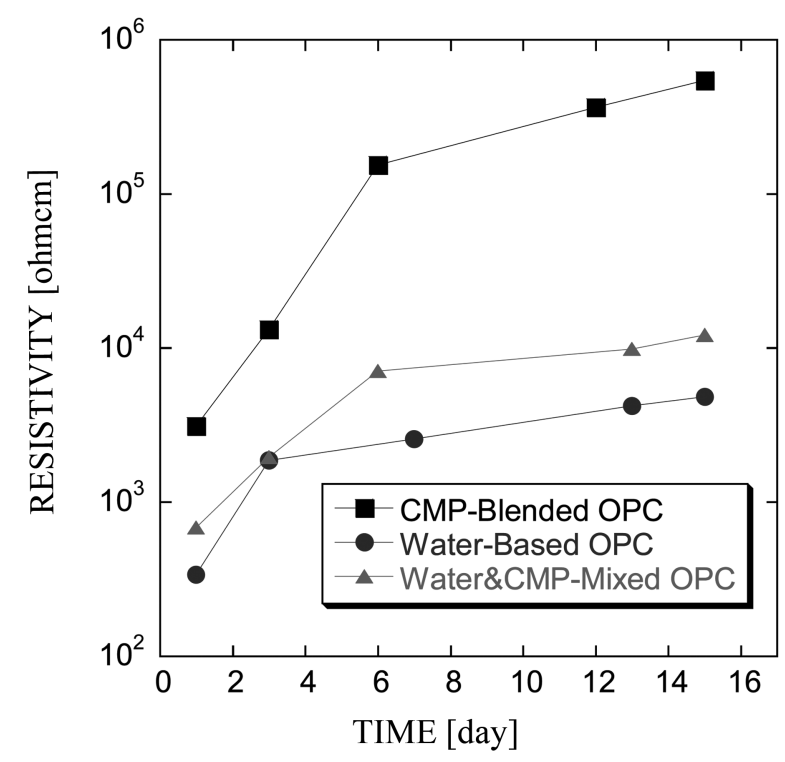

Fig. 5. Resolved resistivity calculated as a function of the composition and hydration time.

as a function of the hydration time and composition. As the hydration time increased, the overall resistance increased regardless of the composition of the CMP-blended cements. The high frequency impedance was attributed to the bulk responses, i.e. ionic transport along the porous phase filled with a pore solution, which is typically a strong alkaline solution with a $\mathrm{pH}$ value near 13 . The hydration process continuously produces calcium-silicate-hydrate (C-S-H) phases along with an increase in the solid fractions of the high resistivity materials. As shown in Fig. 5, the analyzed resistivity was summarized as a function of the hydration time and composition. The resistivity of the CMP-blended cements without tap water (A1) were significantly higher than that of the OPC cements mixed with tap water only (R1) and that of the OPC mixed with both tap water and the CMP slurry (M1).

As shown in Fig. 6, the hydration changes the electrical behavior and dielectric responses, as monitored using the frequency-dependent capacitance bode plots. Furthermore, the capacitance exhibited a frequency-dependent variation: at a high frequency, the capacitance was in the range of tens of picofarads, and thereafter the capacitance continued to increase to the range of microfarads. The millifarad range of capacitance is characteristic of the ionic transfer between the electronic conductors and ionic solution. As noticed in Fig. 6, hydration continues to decrease the capacitance due to the continuously evolving solid phases intruding into the liquid phases located within the porous paths using the abovementioned C-S-H products. The time-dependent resistance and capacitance indicate that the hydration process progresses as long as the relative humidity is maintained under $100 \%$. Furthermore, the high frequency capacitance decreases with increases in the hydration time. As the hydration proceeds, the capacitance decreases and the corresponding time constants change to higher values: the semi-circle begins to be formed at a lower frequency as the hydration time increases.

The high frequency information is concurrent with the continuously evolving microstructure where the hydration in the cements continues to produce solid phases, such as electrically insulating phases, e.g. calcium-silicate-hydrate (C-S-H). Typically, the pore solution (obtained from the OPC pastes) includes high concentrations of positive and negative ions such as $\mathrm{Na}^{+}, \mathrm{K}^{+}$, and $\mathrm{OH}^{-}$along with a high conductivity. The pore structure of the highly conductive ionic solution is subjected to interactions with the solid phases made from C-S-H products and solid phases; hence, the pore structure becomes more tortuous in terms of pore length and size as the hydration or equivalent setting proceeds. The frequency-dependent IS is believed to allow the electrical monitoring of the self-setting characteristics that occur in cement-based materials.

Considering the high frequency bulk-based responses, the cement-based materials can be regarded as 3-3 interconnected materials according to the Electrocomposite Theory explained by Newnham's group. ${ }^{7)}$ The solid component is made from either the initial OPC cements or $\mathrm{Al}_{2} \mathrm{O}_{3}$ particles,

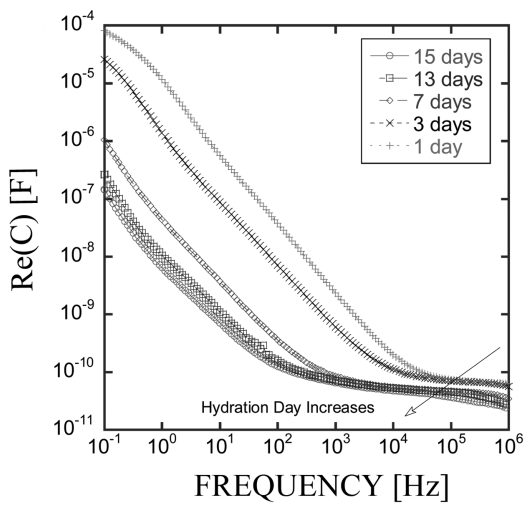

(a)

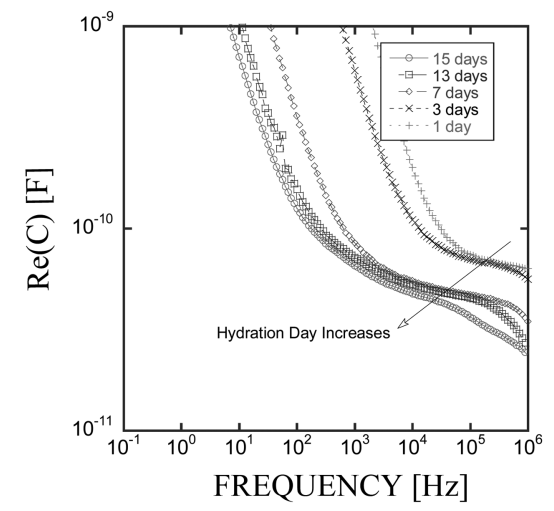

(b)

Fig. 6. Capacitance bode plot as a function of the frequency in the CMP-blended cements. (b) is an enlargement of (a) in the high frequency regime. 
and the hydration products. The solid phase is highly resistive or insulating compared with that of the pore solution. Consequently, the conductive pore solution predominantly controls the electrical/dielectric hydration response in the cement-based materials. As hydration continues, the average pore size decreases continuously and the interconnected paths in the capillary pores become narrower and longer. In particular, the current CMP slurry includes insulating $\mathrm{Al}_{2} \mathrm{O}_{3}$ nanocrystals, and the $\mathrm{Al}_{2} \mathrm{O}_{3}$ appears to block the pore solution and accelerate the hydration process, thereby functioning as a nucleating agent. This explanation is corroborated by the considerably higher resistivity found in the cement-CMP mixtures (see Fig. 6). In the cement-based materials, the electrical conduction is determined by the overall combination of the pore structure and pore solution. In the CMP-blended cements, the ionic solution and changing microstructure can be assumed to be quite similar to those of the OPCs. ${ }^{3)}$ The electrical conduction in both systems is dominated by the amount, size, and distribution of the pores in the cement-based materials. The resultant conductivity can be described using the proposed Electrocomposite Theory. ${ }^{7)}$ The apparent conductivity is given by:

$$
\sigma_{t}=\Sigma \sigma_{i} \beta_{i} \phi_{i}=\sigma_{s} \beta_{s} \phi_{s}+\sigma_{o} \beta_{o} \phi_{o}
$$

where $\sigma_{t}$ is the apparent conductivity, $\sigma_{i}$ is the conductivity of phase $i, \beta_{i}$ is the interconnectivity of phase $i$, and $\phi_{i}$ is the volume fraction of phase $i$. The subscripts $s$ and o denote the solid and liquid phases, respectively. Considering the high contribution of the ionic liquid component, Equation (1) is simplified into:

$$
\sigma_{\mathrm{t}}=\sigma_{\mathrm{o}} \beta_{\mathrm{o}} \phi_{\mathrm{o}}
$$

That is, the overall conduction is dependent on the porosity and interconnectivity of the porous regions filled with the high conductivity pore solution within the CMP-blended cements. As shown in Fig. 5, the time-dependent hydration is closely associated with a significant increase in resistivity. Therefore, the evolving microstructures of the solid and liquid phases continue to decrease the amount of pore solution in order to become tortuous in terms of the porous components as the hydration progresses for all compositions of the CMP-blended and OPC cements. In particular, the resistivity of the CMP-blended cements is considerably higher than that of OPCs. This result is associated with two features of the blended cements: the $\mathrm{Al}_{2} \mathrm{O}_{3}$ particles accelerate the hydration process, which leads to a significant decrease in the porous phase, and the more tortuous (equivalently less interconnective) microstructure of the CMPblended cements, which leads to a decrease in the conductivity due to the smaller interconnectivity and porosity values, according to Eq. (2).

Hydration in cement-based materials is closely related to the reaction products formed through the consumption of the liquid phases. In cement hydration, the continuous chemical reaction hampers effective ionic conduction due to the continuous growth in the hydration products. This concept was simulated in terms of the microstructural parameters obtained from the cement products, i.e. through both narrower paths and thicker C-S-H products. In these simulations, the resultant resistance increased with the increasing barrier thickness and decreasing hole diameter due to geometrical constrictions in the ionic conduction in the pore solution. ${ }^{8,9)}$ The combined effects of the two pore sizes and thicknesses in the C-S-H phases cause them to be more tortuous, which leads to great difficulty in the formation of interconnected pore solutions. The addition of the CMP slurry appears to accelerate the abovementioned hydration process.

The physical properties of the cement-based materials, i.e. electrical resistivity, creep tests, and compressive strengths, depend critically on their pore network structure. ${ }^{10)}$ The current characterization of the CMP-blended cement mixtures indicates that the electrical impedance information is consistent with the enhanced compressive strengths reported by Lee et al. ${ }^{2)}$ That is, the inorganic nanocrystal components appear to form a higher fraction of the hydration products compared with that resulting from the OPC only.

This work reports the preliminary information of the effect of the CMP slurry on the setting phenomena in cement-based materials and OPCs in particular. The proposed concept should be verified by subsequent microstructure image analyses and using both mercury intrusion porosimetry and compressive strength tests. In this work, the frequency-dependent IS demonstrated its usefulness as a uniquely non-destructive probing technique for the quantitative in situ monitoring of the hydration process involving a time-dependent change in the microstructure using the CMP slurry as an acceleration agent. Contrary to the destructive features of the BET measurements, electron microscopy, and mercury intrusion porosimetry, the frequency-dependent IS demonstrated its effectiveness in monitoring the hydration process involving the CMP slurry materials. A forthcoming publication will report the chemical and microstructural issues in more depth.

\section{Conclusions}

The frequency-dependent IS was proven to be a nondestructive approach for the electrical monitoring of cement-based materials in both single and composite systems. The impedance spectra of the CMP-blended cement mixtures reflected the simultaneous measurement of the bulk and electrode based responses regarding the resistive and capacitive components. The presence of the CMP nanocrystals accelerated the hydration process that occurs in the OPCs, which led to a significant increase in the resistivity of the CMP-based OPC mixtures. These effects were a result of a significant change in three-dimensional pore structure with an emphasis on the pore size distribution and interconnectivity. The presence of the $\mathrm{Al}_{2} \mathrm{O}_{3}$ particles in 
the CMP slurry appeared to block the interconnected porous phase and accelerate the hydration process due to the beneficial effect of the alumina particles in the formation of the C-S-H materials.

\section{Acknowledgments}

The author is grateful to K.-R. Lee and Y.-H. You for their assistance in preparing the manuscript. This work was supported by a 2009 Hongik University Research Fund.

\section{REFERENCES}

1. M. D. G. de Luna, Warmadewanthi, and J. C. Liu. "Combined Treatment of Polishing Wastewater and Fluoridecontaining Wastewater from a Semiconductor Manufacturer," Colloid Surf., A347 64-8 (2009).

2. T.-C. Lee, K.-L. Lin, X.-W. Su, and K.-K. Lin, "Recycling CMP Sludge as a Resource in Concrete," Tzen-Chin Construt. Build. Mater., 30 243-51 (2012).

3. B. J. Christensen, R. T. Coverdale, R. A. Olson, S. J. Ford, E. J. Garboczi, H. M. Jennings, and T. O. Mason, "Impedance Spectroscopy of Hydrating Cement-Based Materials: Measurement, Interpretation, and Application," J. Am. Ceram. Soc., 77 [11] 2789-804 (1994).

4. P. Gu, P. Xie, J. J. Beaudoin, and R. Brousseau, "A. C. Impedance Spectroscopy (I): A New Equivalent Circuit
Model for Hydrated Portland Cement Paste," Cement Concrete Res., 22 833-40 (1992).

5. P. Xie, P. Gu, Z. Xu, and J. J. Beaudoin, "A Rationalized A. C. Impedance Model for Microstructural Characterization of Hydrating Cement Systems," Cement Concrete Res., 23 359-67 (1993).

6. N. Bonanos, B. C. H. Steele, E. P. Butler, J. R. Macdonald, W. B. Johnson, W. L. Worrell, D. D. Macdonald and M. C. H. MaKubre, E. Barsoukov, B. E. Conway, and N. Wagner, "Impedance Spectroscopy Theory, Experiment, and Applications," pp. 205-537, Ed. by E. Barsoukov and J. R. Macdonald, Wiley, New York, 2005.

7. D. S. McLachlan, M. B. Blazkiewicz, and R. E. Newnham, "Electrical Resistivity of Composites," J. Am. Ceram. Soc., 73 [8] 2187-203 (1990).

8. S. J. Ford, J.-H. Hwang, J. D. Shane, R. A. Olson, G. M. Moss, H. M. Jennings, and T. O. Mason, "Dielectric Amplification in Cement Pastes," Adv. Cement Based Mater., 5 41-48 (1997).

9. P. R. Camp and S. Bilotta, "Dielectric Properties of Portland Cement Paste as a Function of Time since Mixing," $J$. Appl. Phys., 66 6007-13 (1989).

10. R. I. A. Malek, D. M. Roy, and Y. Fang, "Pore Structure, Permeability, and Chloride Diffusion in Fly Ash-And SlagContaining Pastes and Mortars," MRS Proceedings, 137 403-10 (1988). 